\title{
A.O. Ifesanya \\ Proximal Focal Femoral Deficiency in Ibadan B.A. Omololu a Developing Country's Perspective and a Review of the Literature
}

Received: 28 February 2011 Accepted: 9 June 2011

A.O. Ifesanya ( $\varangle)$ B.A. Omololu Department of Orthopaedics \& Trauma, University College Hospital, Ibadan. Nigeria. Tel: +2347030172174 e-mail: lekeifesanya@yahoo.com

\begin{abstract}
Proximal focal femoral deficiency (PFFD) is a rare but often severe abnormality of the lower limb which poses a significant challenge to effective treatment. We reviewed 21 patients with 23 cases of PFFD treated in our centre in the 14-year period from 1997 to 2010. The male:female ratio was $1: 2$ and the right femur was more commonly involved. Coincident congenital malformations were identified in four patients (19\%), all involving the limbs. Conservative management was
\end{abstract}

employed for all our patients due to non-availability of limb lengthening facilities. The cultural aversion to amputation in our environment makes it difficult to employ that option of treatment.

Proximal focal femoral deficiency in Ibadan a developing country's perspective and a review of the literature.

Keywords: Proximal focal femoral deficiency, congenital malformations, 1 i mb malformations, lower limb anomalies, Nigeria.

\section{Introduction}

Proximal focal femoral deficiency (PFFD) otherwise known as proximal femoral dysplasia is said to be a rare congenital deformity with a reported incidence of 1 per 50,000 live births and various maternal conditions being implicated ${ }^{1,2,3,4,5}$. Like many other congenital and transverse deficiencies, PFFD includes a broad spectrum of defects. Minor forms present as hypoplasia of the femur, whereas severe involvement may result in femoral agenesis ${ }^{2,3}$.

The management of this condition poses a lot of challenge to the Orthopaedic Surgeon because of the peculiar sociocultural circumstances of our society. Aspects of recognised management protocols e.g. amputation also evokes significant emotional and psychological disturbances in our environment.

This report aims to draw attention to the existence of this condition in our environment as it is yet to be reported in the West African subregion. We present Some cases of PFFD as seen at our centre and a review of the literature to elaborate on the management problems encountered in this supposedly rare disease. 
Figures 1 to 4 show examples of different variants of the disease as seen in this environment.

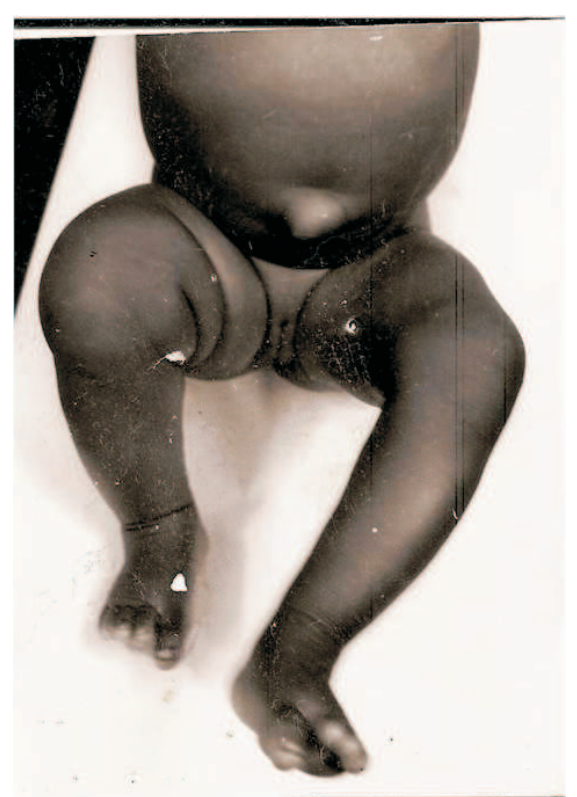

Figure 1 Clinical picture showing a girl with right sided PFFD. Note the extra skin creases; the shortening of the right thigh is not obvious here.

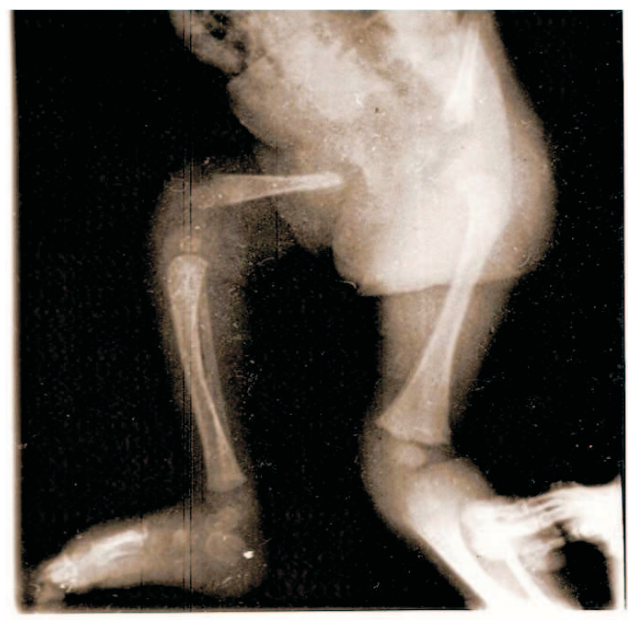

Figure 2 Radiograph showing right-sided PFFD. Note absence of a femoral head but presence of an acetabulum (type C).

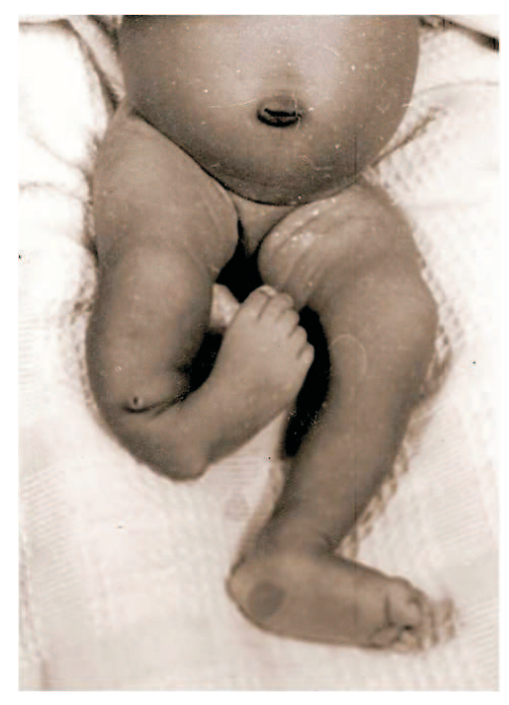

Figure 3 Clinical picture showing a girl with rightsided PFFD. There is an associated right tibial pseudoarthrosis which explains the shortened and deformed right leg.

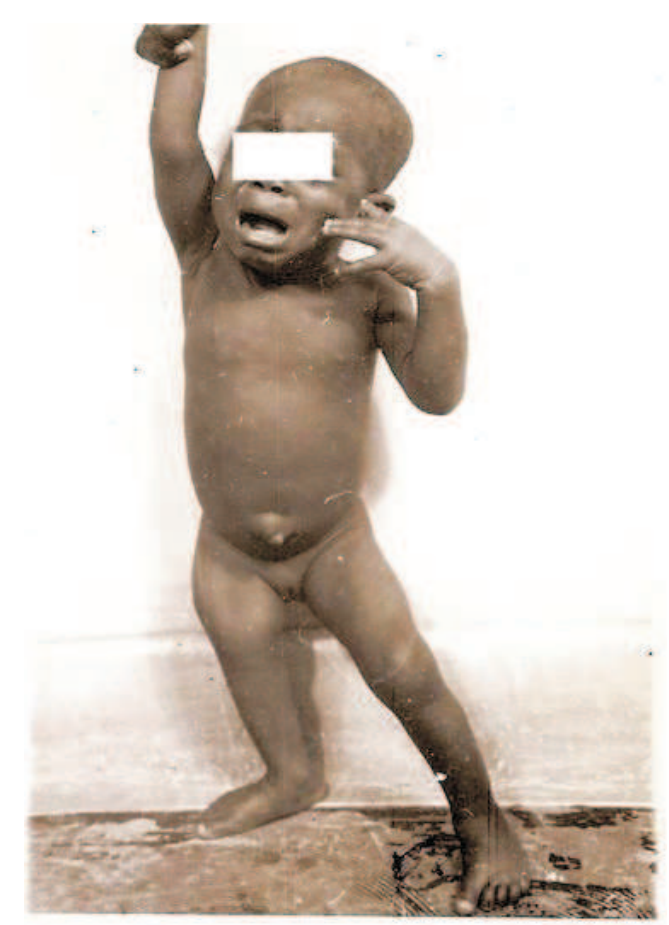

Figure 4 This clinical picture shows a female child with right-sided PFFD. As she stands, the discrepancy in length of the lower limbs is obvious. 
Figure 5 shows a patient already standing with a shortened limb while Figure $\mathbf{6}$ is a radiograph of the hips and thighs of the same patient showing absent femoral head and acetabulum bilaterally.

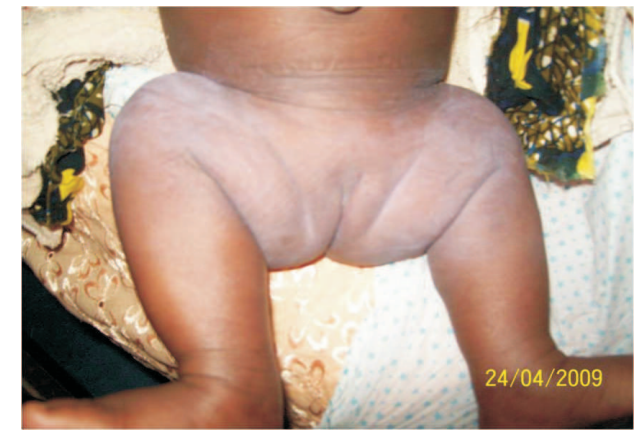

Figure $5 \mathrm{~A}$ female infant with bilateral PFFD. Note the severe shortening of both thighs and externally rotated legs.

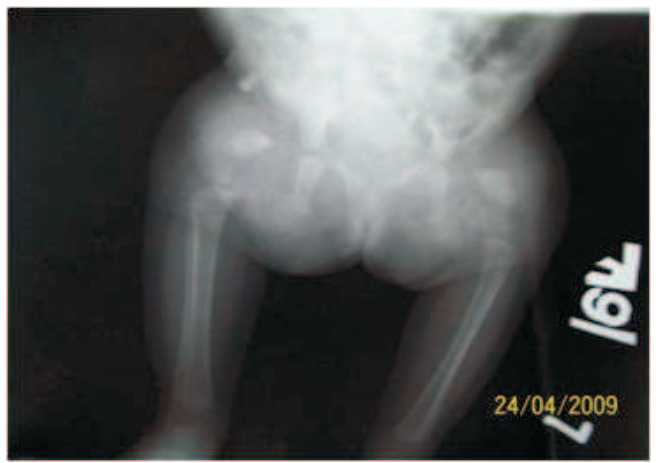

Figure 6 Radiograph of the infant shown in figure 5 above. There is neither femoral head nor acetabulum on either side (type D).

\section{Literature Review}

Proximal focal femoral deficiency is characterised by considerable shortening in association with a stable hip which is freely mobile from a position of some fixed flexion and lateral rotation ${ }^{2,3}$. Most commonly, PFFD consists of a partial skeletal defect in the proximal femur with a variably unstable hip joint; associated anomalies, which include fibular hemimelia and agenesis of the cruciate ligaments of the knee especially when there is bilateral involvement ${ }^{4,5,6,7,8}$. Other congenital anomalies reported in association with PFFD include clubfoot, congenital heart anomalies, spinal dysplasia and facial dysplasias ${ }^{4,5}$ and most patients are said to have other congenital anomalies. Nineteen percent of our series had associated anomalies all of which involved the limbs.

The radiological features usually are a short femur associated with apparent absence of the proximal third of the femoral shaft, trochanteric area, and neck. The radiological findings are therefore not compatible with the clinical findings; for were such a defect present, the hip would be totally unstable and would lack fixed deformity ${ }^{5}$. The inference to be drawn is that there is continuity between the femoral head and the proximal end of the shortened femoral shaft. The intervening transradiant area is occupied by a cartilaginous model in which ossification is delayed.

Embryologically, it is accepted that the ilium and proximal end of the femur develop from a common cartilaginous anlage in the human. Observations confirm that the limb develops in a proximo-distal direction in such a way that in fetuses in which there is no acetabulum, no femoral head develops. Sclerotome subtraction have been offered as an explanation for various limb-reduction deformities? . This theory proposes that injury to the position of the neural crest that forms the precursor of the peripheral sensory nerves for the fourth and fifth lumbar vertebra results in a proximal femoral dysplasia. Among the substances postulated as teratological agents are irradiation, anoxia, ischaemia, mechanical or thermal injury, bacterial toxins, viral infection, chemicals and hormones ${ }^{4}$. However, to date only thalidomide taken by the mother between the fourth and sixth week after conception, has been shown to be a definite cause in humans $\mathrm{s}^{4,9,10}$.

We have not been able to associate any teratological agents with any of our cases as no parent volunteered useful information on this.

Aitken's four-part (A, B, C, D) classification is one of the earliest attempts to provide a systematic taxonomy of this condition ${ }^{1}$.

The rational management of PFFD is dependent upon the identification of those likely to develop instability, so that measures to prevent this occurring may be taken before continuity is $\operatorname{los}^{2,3,4,5}$. The major problems are limb length inequality and variable inadequacy of the proximal femoral musculature and hip joint. Treatment is indicated and ranges from amputation and prosthetic rehabilitation to limb salvage, lengthening, and hip reconstruction.

Until the early 1960s, treatment for PFFD at the St Louis Shrines Hospital was essentially conservative? The natural history of the particular variant and limitations of surgical reconstruction must be considered. Most often no surgical reconstruction of any kind is indicated. Most authors agree that bilateral PFFD is best treated without surgery. The patients learn to accept their short stature and are quite functional.

The goal of surgical intervention is functional ambulating, with many procedures specifically designed to facilitate optimal prosthetic usage. Stability of the hip is important in determining treatment. In patients with both a femoral head and acetabulum (Aitken classes A and B), many authors 
have recommended surgery to establish continuity between the femoral head and the femur, but this may be technically difficult if there is little bone stock to work with in the proximal femur ${ }^{1,2,3,4,5,6,7}$. For this reason, surgery is best delayed until there is adequate ossification of the femoral head and proximal metaphysis. In some patients, the femur is so short that a simultaneous knee fusion is performed, creating a one-bone leg. This would be necessary in our patient in Fig 3 with congenital absence of the distal femur. Although the radiographic picture may be improved, with the correction of the proximal pseudoarthrosis, it remains to be shown that function is improved. In fact, many patients treated nonoperatively have good motion and reasonably good function. Stabilizing the proximal pseudoarthrosis may diminish the overall range of motion of the hip. For less severe PFFD, hip reconstruction is limited to a valgus osteotomy that improves biomechanical alignment for severe coxa vara. Care must be taken not to damage the proximal femoral epiphyseal plate in these children who already have problems with diminished growth of the femur.

For severe deformities in which there is no femoral head or acetabulum (Aitken classes C and D), most authors recommend that no attempt be made at hip reconstruction, although there are notable exceptions ${ }^{1,2,3,4,5}$. King recommends iliofemoral fusion, which requires a simultaneous Chiari osteotomy to create a suitable bony bed to receive the small femoral remnant leaving the knee joint to assume the function of the hip joint ${ }^{11,12,13,14}$. Fixen and Lloyd-Roberts also used the technique, with additional bone graft to ensure fusion ${ }^{2}$. Although this technique eliminates the hip instability, it may severely limit mobility of the limb. Even with a certain amount of instability, the knee generally functions as a hinge providing flexion and extension only. Rotation and abduction are lost after iliofemoral arthrodesis.
Surgical limb lengthening, with or without contralateral shortening, should be considered only in selected patients. In 1982, Herring and Coleman suggested $10-12 \mathrm{~cm}$ as the maximum amount of lengthening possible in a single long bone with congenital deficiency and combined with contralateral shortening, $17 \mathrm{~cm}$ as the maximum amount of inequality that could be corrected ${ }^{15}$. They recommend limb lengthening only in the femur with over $60 \%$ of predicted femoral length or less than $17 \mathrm{~cm}$ of projected shortening; other prerequisites for lengthening were hip stability and a stable, plantigrade foot. Gillespie and Torode, using Wagner's technique of leg lengthening, suggested that lengthening be considered for femurs that are at least $60 \%$ of normal length ${ }^{3,16,17}$. The Ilizarov method of lengthening, using thin-wire circular external fixators, may extend these limits. Regardless of technique, limb lengthening in patients with PFFD is difficult, with the ever-present danger of knee and hip subluxation. For predicted discrepancies greater than $12-14 \mathrm{~cm}$, lengthening may be performed in two stages: one at 8 or 9 years and a second during the early teens ${ }^{15,16,17}$. Depending on the predictions of the patient's overall based on the normal leg, a contralateral epiphyseodesis may be indicated ${ }^{18}$.

\section{Conclusion}

The spectrum of management options available to the surgeon for the care of the patient with PFFD is as wide as the deformities associated with the entity. However, in this environment with the limited resources and the non-availability of equipments for limb lengthening, one is confined to the option of conservative management, arthrodesis, or amputation in severe cases like in Fig 3.

However, in this environment with our socio-cultural aversion to amputation, it is an arduous task convincing parents about an amputation in a limb that may look normal to them. In all our cases so far, we have employed conservative management methods while the majority are still being followed up with the possibility of limb lengthening in the near future. 
1. Aitken GT. Proximal femoral focal deficiency: definition, classification and management. In: Aitken GT. Ed. Proximal Femoral Focal Deficiency: a congenital anomaly. Washington DC; National Academy of Sciences, 1969.

2. Fixen JA, Lloyd-Roberts GC. The natural history and early treatment of proximal femoral dysplasia. J Bone Joint Surg 1974;56-B:86-95.

3. Gillespie R, Torode IP. Classification and management of congenital abnormalities of the femur. J Bone Joint Surg 1983;65B:5576-5578.

4. Epps HE. Current concepts reviiew: proximal femoral focal deficiency. J Bone Joint Surg 1983;65-A:867-870.

5. Lange DR, Schoenecker PL, Baker CL. Proximal femoral focal deficiency: treatment and classification in fortytwo cases. Clin Orthop 1978;135:15-25.

6. Aitken GT. Congenital short femur with fibular hemimelia. J Bone Joint Surg 1974;56-A:1299-1316.
7. Amstutx HC, Wilson PD Jr. Dysgenesis of the proximal femur (coxa vara) and its surgical management. J Bone Joint Surg 1962;44-A:1-24.

3. Johnson CE. Congenital short femur with coxa vara. Orthopedics 1983;6:892896.

9. McCredie J. Sclerotome subtraction: a radiological interpretation of reduction deformities of the limbs. In: Bergsma D, Lowry RB. Eds. E m b r y o log y a n d Pathogenesis and Prenatal Diagnosis. New York. Alan R Liss, 1977;65-67.

10.Hamanishi C. Congenital short femur, clinical, genetic, and epidemiological comparison of the naturally occurring condition with that caused by thalidomide. J Bone Joint Surg 1980;62B:307-320.

11. King RE. Some concepts of proximal femur focal deficiency. In: Aitken GT. Ed. Proximal Femoral Focal Deficiency: a symposium. Washington DC. National Academy of Sciences, 1969.

12. King RE. Surgical correction of proximal femoral focal deficiency. Inter-clin Info Bull 1965:4-16.
13. King RE. Proximal femoral focal deficiency. In: Tronco RG. Ed. Surgery of the Hip Joint. Philadelphia. Lea and Febiger, 1973;126-156.

14. Steel HH, Lin PS, Betz RR, et al. Iliofemoral fusion for proximal femoral focal deficiency. J Bone Joint Surg 1987;69-A:837-843.

15. Herring JA, Coleman SS. Femoral lengthening. J $\mathrm{P}$ ediatric Orthop 1982;24:432-435.

16. Torode IP, Gillespie R. Rotation plasty of the lower limb for congenital defects of the femur. J Bone Joint Surg 1983;65-B:569-573.

17. Hood RW, Riseborough EJ. Lengthening of the lower extremity by Wagner method: a review of the Boston Children's Hospital experience. J Bone Joint Surg 1987;69-A:699-705.

18. Menelaus MB. Correction of leg length discrepancy by epiphyseal arrest. J Bone Joint Surg 1966;48-B:336339. 